\title{
Improved control of delayed measured systems
}

\author{
Jens Christian Claussen and Heinz Georg Schuster \\ Institut für Theoretische Physik der Universität Kiel, 24098 Kiel, Germany
}

(Revised: July 29, 2004)

\begin{abstract}
In this paper we address the question how the control of delayed measured chaotic systems can be improved. Both unmodified OGY control and difference control can be successfully applied only for a certain range of Lyapunov numbers depending on the delay time. We show that this limitation can be overcome by at least two classes of methods, by rhythmic control and by the memory methods linear predictive logging control (LPLC) and memory difference control (MDC).
\end{abstract}

\section{INTRODUCTION}

Delay is a generic problem in the control of chaotic systems. The effective delay time $\tau$ in any feedback loop is the sum of at least three delay times, the duration of measurement, the time needed to compute the appropriate control amplitude, and the response time of the system to the applied control. The latter effect appears especially when the applied control additionally has to propagate through the system. These response time may extend to one or more cycle lengths [1].

For the formal situation of fixed point stabilization in time-continuous control, the issue of delay has been investigated widely in control theory, dating back at least to the Smith predictor 22. This approach mimics the, yet unknown, actual system state by a linear prediction based on the last measurement. Its time-discrete counterpart (LPLC) discussed below allows to place all eigenvalues of the associated linear dynamics to zero, and always ensures stability. The (time-continuous) Smith predictor with its infinite-dimensional initial condition had to be refined 3, 4], giving rise to the recently active fields of model predictive control [5]. For fixed point stabilization, an extension of permissible latency has been found for a modified proportional-plus-derivative controller [6].

If one wants to stabilize the dynamics of a chaotic system onto an unstable periodic orbit, one is in a special situation. In principle, a proper engineering approach could be to use the concept of sliding mode control 7], i. e. to use a co-moving coordinate system and perform suitable control methods within it. However, this requires the quite accurate knowledge of the whole trajectory (and the direction of the stable manifold) with the respective numerical or experimental costs.

Therefore direct approaches have been developed by explicitely taking into account either a Poincaré surface of section [8] or the explicit periodic orbit length [9]. This field of controlling chaos, or stabilization of chaotic systems, by small perturbations, in system variables [10] or control parameters [8], emerged to a widely discussed topic with applications in a broad area from technical to biological systems. Especially in fast systems [11, 12] or for slow drift in parameters [13, 14], difference control methods have been successful, namely the time-continuous Pyragas scheme 9], ETDAS 11], and time-discrete difference control [15].
Like for the control method itself, the discussion of the measurement delay problem in chaos control has to take into account the special issues of the situation: In classical control applications one always tries to keep the control loop latency as short as possible. In chaotic systems however, one wants to control a fixed point of the Poincare iteration and thus has to wait until the next crossing of the Poincaré surface of section, where the system again is in vicinity of that fixed point.

The stability theory and the delay influence for timecontinuous chaos control schemes has been studied extensively 16, 17, 18, 19, 20], and an improvement of control by periodic modulation has been proposed in 21]. For measurement delays that extend to a full period, however no extension of the time-continuous Pyragas scheme is available.

In this paper we investigate time-discrete control schemes and focus on the question what limitations occur if one applies OGY control [8] or difference feedback [15] in the presence of time delay, and what strategies can be used to overcome these limitations. We show how the measurement delay problem can be solved systematically for OGY control and difference control by rhythmic control and a memory method and give constructive direct and elegant formulas for the deadbeat control in the timediscrete Poincaré iteration. While the predictive control method LPLC presented below for OGY control has a direct correspondence to the Smith predictor and thus can be reviewed as its somehow straightforward implementation within the unstable subspace of the Poincaré iteration, this prediction approach does not guarantee a stable controller for difference control. However, within a class of feedback schemes linear in system parameters and system variable, there is always a unique scheme where all eigenvalues are zero, the MDC scheme presented below. The method can be applied also for more than one positive Ljapunov exponent, and shows, within validity of the linearization in vicinity of the orbit, to be free of principal limitations in Ljapunov exponents or delay time. For zero delay (but the inherent 1 period delay of MDC), MDC has been demonstrated experimentally for a chaotic electronic circuit 13] and a thermionic plasma discharge diode 14], with excellent agreement, both of stability areas and transient Ljapunov exponents, to the theory presented here.

The paper is organized as follows. After introducing 
the notation within a recall of OGY control, we give a brief summary what limitations occur for unmodified OGY control; details can be found in 22. In Section III we introduce different memory methods to improve control, of which the LPLC approach appears to be superior as it allows stabilization of arbitrary fixed points for any given delay. The stabilization of unknown fixed points is discussed in Section [V] where we present a memory method (MDC) that again allows stabilization of arbitrary unstable fixed points. As shown in Appendix A for all systems with only one instable Lyapunov number, the iterated dynamics can be transformed on an eigensystem which reduces to the one-dimensional case. The explicit formulas for the case of higher-dimensional subspaces are given in Appendices B and $\mathrm{C}$

\section{CONTROL OF UNSTABLE PERIODIC ORBITS}

\section{A. Ott, Grebogi, Yorke control}

The OGY method given by Ott, Grebogi and Yorke [8] stabilizes unstable fixed points (or unstable periodic orbits utilizing a Poincaré surface of section) by feedback that is applied in vicinity of the fixed point $x^{*}$ of a discrete dynamics $x_{t+1}=f\left(x_{t}, r\right)$.

Thus, for a chaotic flow (or corresponding experiment) one reduces the system dynamics

$$
\dot{\vec{x}}=\vec{F}(\vec{x}, r)
$$

to the discrete dynamics between subsequent Poincaré sections at $t_{0}, t_{1}, \ldots t_{n}$. This description is fundamentally different from a stroboscopic sampling as long as the system is not on a periodic orbit, where the sequence of differences $\left(t_{i}-t_{i-1}\right)$ would show a periodic structure.

If there is only one positive Ljapunov exponent, we can proceed considering the motion in unstable direction only (see Appendix A), i. e. a one-dimensional iterated map. (For 2 or more positive Ljapunov exponents on can proceed in a similar fashion, see App. B and $\mathrm{C}$ )

In OGY control, the control parameter $r_{t}$ is made timedependent. The amplitude of the feedback $r_{t}=r-r_{0}$ added to the control parameter $r_{0}$ is proportional by a constant $\varepsilon$ to the distance $x-x^{*}$ from the fixed point, i. e. $r=r_{0}+\varepsilon\left(x_{t}-x^{*}\right)$, and the feedback gain can be determined from a linearization around the fixed point, which reads, if we neglect higher order terms,

$$
\begin{aligned}
f\left(x_{t}, r_{o}+r_{t}\right)= & f\left(x^{*}, r_{0}\right)+\left(x_{t}-x^{*}\right) \cdot\left(\frac{\partial f}{\partial x}\right)_{x^{*}, r_{0}} \\
& +r_{t} \cdot\left(\frac{\partial f}{\partial r}\right)_{x^{*}, r_{0}} \\
= & f\left(x^{*}, r_{0}\right)+\lambda\left(x_{t}-x^{*}\right)+\mu r_{t} \\
= & f\left(x^{*}, r_{0}\right)+(\lambda+\mu \varepsilon) \cdot\left(x_{t}-x^{*}\right)
\end{aligned}
$$

The second expression vanishes for $\varepsilon=-\lambda / \mu$, that is, in linear approximation the system arrives at the fixed point at the next time step, $x_{t+1}=x^{*}$. The uncontrolled system is assumed to be unstable in the fixed point, i. e. $|\lambda|>1$. The system with applied control is stable if the absolute value of the eigenvalues of the iterated map is smaller than one,

$$
\left|x_{t+1}-x^{*}\right|=\left|(\lambda+\mu \varepsilon) \cdot\left(x_{t}-x^{*}\right)\right|<\left|x_{t}-x^{*}\right|
$$

Therefore $\varepsilon$ has to be chosen between $(-1-\lambda) / \mu$ and $(+1-\lambda) / \mu$, and this interval is of width $2 / \mu$ and independent of $\lambda$, i.e. fixed points with arbitrary $\lambda$ can be stabilized. This property however does not survive for delayed measurement. One has to develop further control strategies to cover this class of systems.

\section{B. Delay matching in experimental situations}

Before discussing the time-discrete reduced dynamics in the Poincaré iteration, it should be clarified how this relates to an experimental control situation. On the first glance, the time-discrete viewpoint seems to correspond only to a case where the delay (plus waiting time to the next Poincaré section) exactly matches the orbit length, or a multiple of it. The generic experimental situation however comes up with a non-matching delay. Application of all control methods discussed here requires to introduce an additional delay, usually by waiting for the next Poincaré crossing, so that measurement and control are applied without phase shift at the same position of the orbit. In this case the next Poincaré crossing position $x_{t+1}$ is a function of the values of $x$ and $r$ at a finite number of previous Poincaré crossings only, i. e. it does not depend on intermediate positions. Therefore the (a priori infinite-dimensional) delay system reduces to a finite-dimensional iterated map.

If the delay (plus the time of the waiting mechanism to the next Poincaré crossing) is not matching the orbit length, the control schemes may perform less efficient. Even for larger deviations from the orbit, the time between the Poincaré crossings will vary only marginally, thus a control amplitude should be available in time. In practical situations therefore the delay should not exceed the orbit length minus the variance of the orbit length that appears in the respective system and control setup.

In a formal sense, the Poincaré approach ensures robustness with respect to uncertainties in the orbit length, as it always ensures a synchronized reset of both trajectories and control. Between the Poincaré crossings the control parameter is constant, the system is independent of everything in advance of the last Poincaré crossing. It is solely determined by the differential equation (or experimental dynamics). Thus the next crossing position is a well defined iterated function of the previous one.

This is quite in contrast to the situation of a delaydifferential equation (as in Pyragas control), which has an infinite-dimensional initial condition it 'never gets rid of'. One may proceed to stability analysis via Floquet theory [25] as investgated for continuous [16] and Poincaré-based 
[23, 26] control schemes. Though a Poincaré crossing detection may be applied as well, the position will depend not only on the last crossing, but also on all values of the system variable within a time horizon defined by the maximum of the delay length and the (maximal) time difference between two Poincaré crossings (due to the non-stroboscopic character). Thus the Poincaré iteration would be a function between two infinite-dynamical spaces. Apart from further mathematical subtleties, for a delay differential equation with fixed delay the major advantage of a Poincaré map, reducing the system dynamics to a low-dimensional system, thus completely breaks down.

For all control schemes discussed within this paper, howvever the additional dimensionality is not a continuous horizon of states, but merely a finite set of values that were measured at the previous Poincaré crossings.

\section{OGY CONTROL: DELAYED CASE, RHYTHMIC AND SIMPLE MEMORY CONTROL}

\section{A. Unmodified control of delayed measured maps}

To illustrate the problem, we recall the simple case where no modification of the OGY scheme is taken into account 22]. For $\tau$ time steps delay, unmodified proportional feedback is applied:

$$
r_{t}=\varepsilon\left(x_{t-\tau}-x^{*}\right)
$$

Without loss of generality, we can choose $r_{t}=0$ if no control is applied, and $x^{*}=0$ in the remainder. Using the time-delayed coordinates $\left(x_{t}, x_{t-1}, x_{t-2}, \ldots x_{t-\tau}\right)^{\mathrm{T}}$ :

$$
\left(\begin{array}{c}
x_{t+1} \\
\vdots \\
\\
\vdots \\
x_{t-\tau+1}
\end{array}\right)=\left(\begin{array}{ccccccc}
\lambda & 0 & \cdots & & \cdots & 0 & \varepsilon \mu \\
1 & 0 & & & & 0 \\
0 & 1 & \ddots & & & \vdots \\
\vdots & & \ddots & & & \\
& & & & \ddots & \\
\vdots & & & & \ddots & 0 & \vdots \\
0 & \cdots & & \cdots & 0 & 1 & 0
\end{array}\right)\left(\begin{array}{c}
x_{t} \\
\vdots \\
\\
\\
\vdots \\
x_{t-}
\end{array}\right)
$$

To ensure stability, one has to fulfill $\left|\alpha_{i}\right|<1$ for all eigenvalues $\alpha_{i}$. The stability area is investigated in [22], yielding a $\tau$-dependent maximal Ljapunov number of

$$
\lambda_{\max }=1+\frac{1}{\tau} .
$$

For $\lambda \geq \lambda_{\max }(\tau)$ no control is possible 22].

\section{B. Rhythmic OGY control}

As pointed out for difference control in the case $\tau=0$ in $15,23,24,27]$, one can eliminate the additional degrees of freedom caused by the delay term. One can restrict himself to apply control rhythmically only every $\tau+1$ timesteps $(\tau+2$ for difference control), and then leave the system uncontrolled for the remaining timesteps. Then $\varepsilon=\varepsilon(t)$ appears to be time-dependent with

$$
\varepsilon(t \bmod \tau)=\left(\varepsilon_{0}, 0, \ldots, 0\right)
$$

and, after $(\tau+1)$ iterations of (5), we again have a matrix as in (5), but with $\lambda^{\tau+1}$ instead of $\lambda$. Equivalently, we can write

$$
x_{t+(\tau+1)}=\lambda^{\tau+1} x_{t}+\varepsilon_{0} \mu x_{t} .
$$

What we have done here, is: controlling the $(\tau+1)$ fold iterate of the original system. This appears to be formally elegant, but leads to practically uncontrollable high effective Lyapunov numbers $\lambda^{\tau+1}$ for both large $\lambda$ and large $\tau$.

Even if the rhythmic control method is of striking simplicity, it remains unsatisfying that control is kept quiet, or inactive, for $\tau$ time steps. Even if the state of the system $x$ is known delayed by $\tau$, one knows (in principle) the values of $x_{t}$ for $t<\tau$, and one could (in principle) store the values $\delta r_{t-\tau} \ldots \delta r_{t}$ of the control amplitudes applied to the system. This can be done, depending on the timescale, by analog or digital delay lines, or by storing the values in a computer or signal processor (there are some intermediate frequency ranges where an experimental setup is difficult).

Both methods, rhythmic control and simple feedback control in every time step, have their disadvantages.

For rhythmic control it is necessary to use rather large control amplitudes, in average $\lambda^{\tau} / \tau$, and noise sums up to an amplitude increased by factor $\sqrt{\tau}$.

For simple feedback control the dimension of the system is increased and the maximal controllable Lyapunov number is bounded by (6).

One might wonder if there are control strategies that avoid these limitations. This has necessarily to be done by applying control in each time step, but with using knowledge what control has been applied between the last measured time step $t-\tau$ and $t$. This concept can be implemented in at least two ways, by storing previous values of $x_{t}$ or previous values of $\delta r_{t}$ (LPLC).

\section{Control using memory for previous states}

The first memory method extends the single delay line to several delay lines, using one control gain coefficient for each:

$$
r_{t}=\varepsilon_{1} x_{t-1}+\varepsilon_{2} x_{t-2}+\ldots+\varepsilon_{n+1} x_{t-n-1}
$$

For $n$ steps memory (and one step delay) the control 
matrix is

$$
\left(\begin{array}{c}
x_{t+1} \\
\vdots \\
\vdots \\
x_{t-n}
\end{array}\right)=\left(\begin{array}{ccccccc}
\lambda & \varepsilon_{1} & \cdots & & & \varepsilon_{n} & \varepsilon_{n+1} \\
1 & 0 & & & & 0 \\
0 & 1 & \ddots & & & \vdots \\
\vdots & & \ddots & & & \\
& & & & \ddots & & \\
\vdots & & & & \ddots & 0 & \vdots \\
0 & \cdots & & \cdots & 0 & 1 & 0
\end{array}\right)\left(\begin{array}{c}
x_{t} \\
\vdots \\
\\
\\
\\
\vdots \\
x_{t-n-1}
\end{array}\right)
$$

with the characteristic polynomial

$$
(\alpha-\lambda) \alpha^{n+1}+\sum_{i=1}^{n} \varepsilon_{i} \alpha^{n-i}
$$

We can choose $\alpha_{1}=\alpha_{2}=\ldots \alpha_{n+2}=-\lambda /(n+2)$ and evaluate optimal values for all $\varepsilon_{i}$ by comparing with the coefficients of the product $\prod_{i=1}^{n+2}\left(\alpha-\alpha_{i}\right)$. This method allows control up to $\lambda_{\max }=2+n$, therefore arbitrary $\lambda$ can be controlled if a memory length of $n>\lambda-2$ and the optimal coefficents $\varepsilon_{i}$ are used.

For more than one step delay, one has the situation $\varepsilon_{1}=0, \ldots, \varepsilon_{\tau-1}=0$. This prohibits the 'trivial pole placement' given above, (choosing all $\alpha_{i}$ to the same value) and therefore reduces the maximal controllable $\lambda$ and no general scheme for optimal selection of the $\varepsilon_{i}$ applies. One can alternatively use the LPLC method described below, which provides an optimal control scheme. One could wonder why to consider the previous state memory scheme at all when it does not allow to make all eigenvalues zero in any case. First, the case of up to one orbit delay and moderately small $\lambda$ already covers many low-period orbits. Second, there may be experimental setups where the feedback of previous states through additional delay elements and an analog circuit is experimentally more feasible than feedback of controlled amplitudes.

In summary, all three methods discussed in this section have principal restrictions, but may be of advantage in special situations especially when a simple setup is required.

\section{Linear predictive logging control (LPLC)}

If it is possible to store the previously applied control amplitudes $r_{t}, r_{t-1}, \ldots$, then one can predict the actual state $x_{t}$ of the system using the linear approximation around the fixed point. That is, from the last measured value $x_{t-\tau}$ and the control amplitudes we compute estimated values iteratively by

$$
y_{t-i+1}=\lambda x_{t-i}+\mu r_{t-i}
$$

leading to a predicted value $y_{t}$ of the actual system state. Then the original OGY formula can be applied, i. e. $r_{t}=$ $-\lambda / \mu y_{t}$. In this method the gain parameters are again linear in $x_{t-\tau}$ and all $\left\{r_{t^{\prime}}\right\}$ with $t-\tau \leq t^{\prime} \leq t$, and the optimal gain parameters can be expressed in terms of $\lambda$ and $\mu$.

In contrast to the memory method presented in the previous subsection, the LPLC method directs the system (in linear approximation) in one time step onto the fixed point. However, when this control algorithm is switched on, one has no control applied between $t-\tau$ and $t-1$, so the trajectory has to be fairly near to the orbit (in an interval with a length of order $\delta / \lambda^{\tau}$, where $\delta$ is the interval halfwidth where control is switched on). Therefore the time one has to wait until the control can be successfully activated is of order $\lambda^{\tau-1}$ larger than in the case of undelayed control.

The LPLC method can also be derived as a general linear feedback in the last measured system state and all applied control amplitudes since the system was measured; choosing the feedback gain parameters so that the linearized system has all eigenvalues zero. The linear ansatz

$$
r_{t}=\varepsilon \cdot x_{t-\tau-i}+\eta_{1} r_{t-1}+\ldots \eta_{\tau} r_{t-\tau}
$$

leads to the dynamics in combined delayed coordinates $\left(x_{t}, x_{t}-1, \ldots, x_{t-\tau}, r_{t-1}, \ldots, r_{t-\tau}\right)$

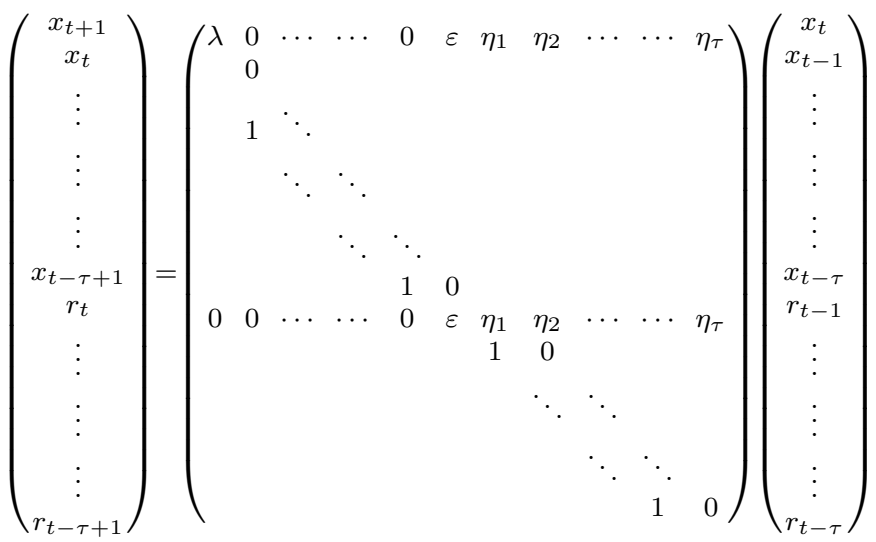

giving the characteristic polynomial

$$
\begin{aligned}
0=-\alpha^{\tau} & \left(\alpha^{\tau+1}+\alpha^{\tau}\left(-\lambda-\eta_{1}\right)\right. \\
& +\alpha^{\tau-1}\left(\lambda \cdot \eta_{1}-\eta_{2}\right)+\alpha^{\tau-2}\left(\lambda \cdot \eta_{2}-\eta_{3}\right) \\
& \left.\ldots+\alpha^{1}\left(\lambda \cdot \eta_{\tau-1}-\eta_{\tau}\right)+\left(\lambda \cdot \eta_{\tau}-\varepsilon\right)\right) .
\end{aligned}
$$

All eigenvalues can be set to zero using $\varepsilon=-\lambda^{\tau+1}$ and $\eta_{i}=-\lambda^{i}$. The general formulas even for more than one positive Lyapunov exponent or multiparameter control are given in Appendix B

\section{E. Nonlinear predictive logging control}

One can also consider a nonlinear predictive logging control (NLPLC) strategy as the straightforward extension to the LPLC method for nonlinear prediction. If the system has a delay of several time steps, the interval where control is achieved becomes too small. However, if 
it is possible to extract the first nonlinearities from the time series, prediction (and control) can be fundamentally improved. In NLPLC the behaviour of the system is predicted each time step by

$$
x_{t+1}=\lambda x_{t}+\frac{\lambda_{2}}{2} x_{t}^{2}+\mu r_{t}+\frac{\mu_{2}}{2} r_{t}^{2}+\nu x_{t} r_{t}+o\left(x_{t}^{3}, \ldots\right)
$$

with using the applied control amplitudes $\left\{r_{t}\right\}$ for each time step. This equation has to be solved for $r_{t}$ using $x_{t+1} \stackrel{!}{=} 0$.

A similar nonlinear prediction method has been described by Petrov and Showalter [28]. They approximate the $x_{t+1}\left(x_{t}, r_{t}\right)$ surface directly from the time series and use it to direct the system to any desired point. Both Taylor approximation or Petrov and Showalter method can be used here iteratively, provided one knows the delay length.

Both approaches could be regarded as a nonlinear method of model predictive control [5], applied to the Poincaré iteration dynamics.

From a practical point of view, it has to be mentioned that one has to know the fixed point $x^{*}$ more accurate than in the linear case. Otherwise one experiences a smaller range of stability and additionally a permanent nonvanishing control amplitude will remain. This may be of disadvantage especially if the fixed point drifts in time (e.g. by other external parameters such as temperature) or if the time series used to determine the parameters is too short.

\section{STABILIZATION OF UNKNOWN FIXED POINTS}

As all methods mentioned above require the knowledge of the position of the fixed point, one may wish to stabilize purely by feeding back differences of the system variable at different times. Without delay, difference feedback can be used successfully for $\varepsilon \mu=-\lambda / 3$, and eigenvalues of modulus smaller than unity of the matrix $\left(\begin{array}{cc}\lambda+\varepsilon \mu & -\varepsilon \mu \\ 1 & 0\end{array}\right)$ are obtained only for $-3<\lambda<+1$, so this method stabilizes only for oscillatory repulsive fixed points with $-3<\lambda<-1$ [15, 27].

Due to the inherent one period delay of MDC, the $\tau$ period delay case of MDC corresponds, in terms of the number of degrees of freedom, to the $\tau+1$ period delay case of LPLC.

\section{A. Unmodified difference control of iterated delayed measured maps}

In the presence of $\tau$ steps delay the linearized dynamics of a simple difference feedback $r_{t}-r_{0}=\varepsilon\left(x_{t-\tau}-x_{t-\tau-1}\right)$ is given by

$$
\left(\begin{array}{c}
x_{t+1} \\
\vdots \\
\\
\vdots \\
x_{t-\tau}
\end{array}\right)=\left(\begin{array}{ccccccc}
\lambda & 0 & \cdots & & 0 & \varepsilon \mu & -\varepsilon \mu \\
1 & 0 & & & & 0 \\
0 & 1 & \ddots & & & \vdots \\
\vdots & & \ddots & & & \\
& & & & \ddots & & \\
\vdots & & & & \ddots & 0 & \vdots \\
0 & \cdots & & \cdots & 0 & 1 & 0
\end{array}\right)\left(\begin{array}{c}
x_{t} \\
\vdots \\
\\
\\
\\
\vdots \\
x_{t-\tau-1}
\end{array}\right)
$$

in delayed coordinates $\vec{y}_{t}=\left(x_{t}, x_{t-1}, \ldots x_{t-\tau-1}\right)$. As we have to use $x_{t-\tau-1}$ in addition to $x_{t-\tau}$, the system is of dimension $\tau+2$, and the lower bound of Lyapunov numbers that can be controlled are found to be 22

$$
\lambda_{\mathrm{inf}}=-\frac{3+2 \tau}{1+2 \tau} .
$$

The controllable range is smaller than for unmodified OGY control, and is restricted to oscillatory repulsive fixed points with $\lambda_{\text {inf }}<\lambda \leq-1$. A striking observation is that inserting $\tau+\frac{1}{2}$ for $\tau$ in eq. (6) leads exactly to the expression in eq. (16) which reflects the fact that the difference feedback can be interpreted as a discretized first time derivative, taken at time $t-\tau-\frac{1}{2}$. For details and stability area see [22].

\section{B. Rhythmic difference control}

To enlarge the range of controllable $\lambda$, one again has the possibility to reduce the dimension of the control process in linear approximation to one by applying control every $\tau+2$ time steps.

$$
\begin{aligned}
x_{t+1} & =\lambda x_{t}+\mu \varepsilon\left(x_{t-\tau}-x_{t-\tau-1}\right) \\
& =\left(\lambda^{\tau+1}+\mu \varepsilon \lambda-\mu \varepsilon\right) x_{t-\tau-1}
\end{aligned}
$$

and the goal $x_{t+1} \stackrel{!}{=} 0$ can be fulfilled by

$$
\mu \varepsilon=-\frac{\lambda^{\tau+1}}{1-\lambda}
$$

One has to choose $\mu \varepsilon$ between $\mu \varepsilon_{ \pm}=-\frac{\lambda^{\tau+1} \pm 1}{1-\lambda}$ to achieve control as shown in Fig. 1. The case $\tau=0$ has already been discussed in [15, 23, 24, 27]. With rhythmic control, there is no range limit for $\lambda$, and even fixed points with positive $\lambda$ can be stabilized by this method.

When using differences for periodic feedback, one still has the problem that the control gain increases by $\lambda^{\tau}$, and noise sums up for $\tau+1$ time steps before the next control signal is applied. Additionally, now there is a singularity for $\lambda=+1$ in the "optimal" control gain given by (17). This concerns fixed points where differences $x_{t}-$ $x_{t-1}$ when escaping from the fixed point are naturally small due to a $\lambda$ near to +1 . 
Here one has to decide between using a large control gain (but magnifying noise and finite precision effects) or using a small control gain of order $\mu \varepsilon_{-}(\lambda=+1)=$ $\tau+1$ (but having larger eigenvalues and therefore slow convergence).
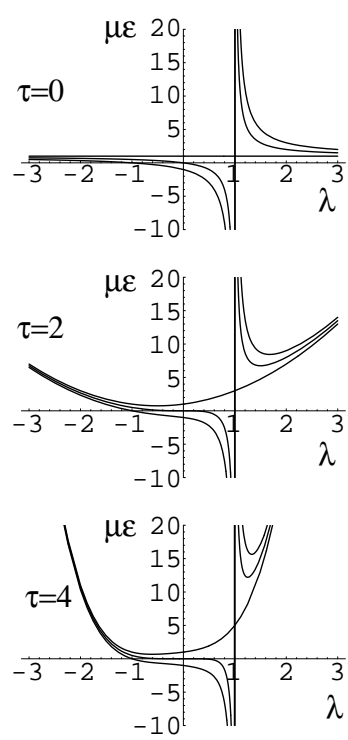
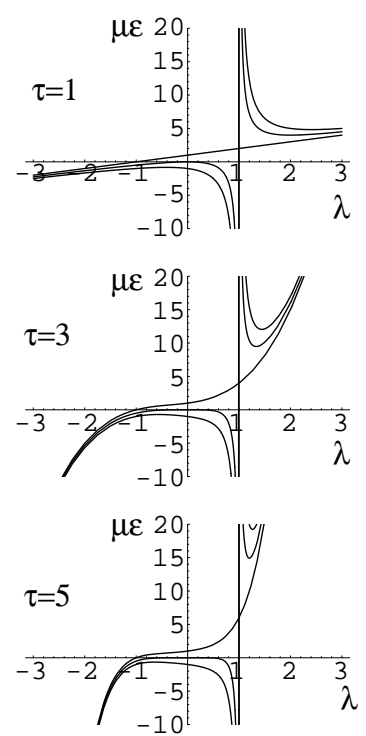

FIG. 1: Periodic difference feedback for $\tau=0,1,2,3,4,5$ : Maximal, optimal and minimal value of $\mu \varepsilon$ for given $\lambda$ to obtain stabilization by control applied every $\tau+2$ time steps.

Two other strategies that have been discussed by Socolar and Gauthier 29] are discretized versions of timecontinuous methods. Control between $\lambda=-(3+R) /(1-$ $R)$ and $\lambda=-1$ is possible with discrete-ETDAS $(R<1)$ $r_{t}=\varepsilon \sum_{k=0}^{\infty} R^{k}\left(x_{t-k}-x_{t-k-1}\right)$ and control between $\lambda=$ $-(N+1)$ and $\lambda=-1$ is acheived with discrete-NTDAS (let $N$ be a positive integer) $r_{t}=\varepsilon\left(x_{t}-\frac{1}{N} \sum_{k=0}^{N} x_{t-k}\right)$. Both methods can be considered to be of advantage even in time-discrete control in the Poincaré section, e.g. if the number of adjustable parameters has to be kept small. Whereas these methods are mainly applied in time-continuous control, especially in analogue or optical experiments, for time-discrete control the MDC strategy described below allows to overcome the limitations in the Lyapunov number.

\section{Memory difference control (MDC)}

One may wish to generalize the linear predictive feedback to difference feedback. In contrary to the LPLC case, the reconstruction of the state $x_{t-\tau}$ from differences $x_{t-\tau-i}-x_{t-\tau-i-1}$ and applied control amplitudes $r_{t-j}$ is no longer unique. As a consequence, there are infinitely many ways to compute an estimate for the present state of the system, but only a subset of these leads to a controller design ensuring convergence to the fixed point.

Under these there exists an optimal every-step control for difference feedback with minimal eigenvalues and in this sense optimal stability. This memory difference control (MDC) method has been demonstrated in an electronic experiment [13] and a plasma diode [14].

To derive the feedback rule, we directly make the linear ansatz

$$
r_{t}=\varepsilon \cdot\left(x_{t-\tau-i}-x_{t-\tau-i-1}\right)+\eta_{1} r_{t-1}+\ldots \eta_{\tau} r_{t-\tau}
$$

leading to the dynamics in combined delayed coordinates

$$
\begin{aligned}
& \left(x_{t}+1, x_{t}, \ldots, x_{t-\tau+1}, r_{t-1}, \ldots, r_{t-\tau+1}\right)^{\mathrm{T}} \\
& \quad=\mathbf{M} \cdot\left(x_{t}, x_{t}-1, \ldots, x_{t-\tau}, r_{t-1}, \ldots, r_{t-\tau}\right)^{\mathrm{T}}
\end{aligned}
$$

with

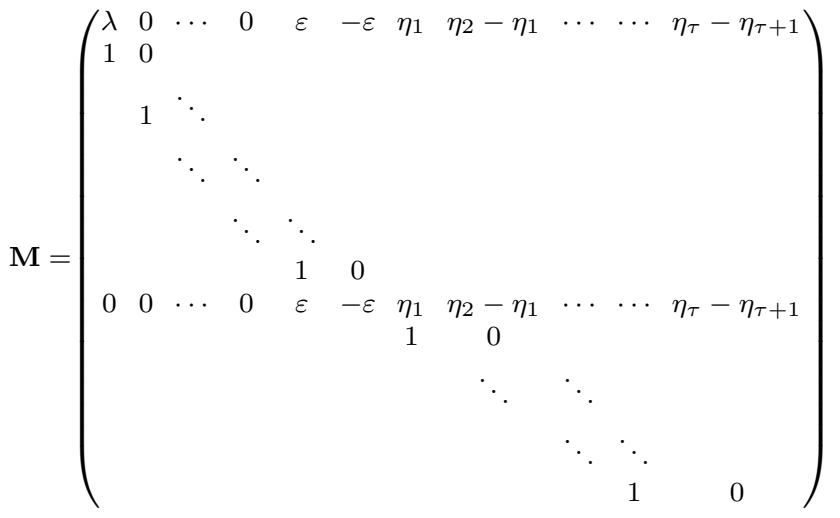

giving the characteristic polynomial

$$
\begin{aligned}
0= & -\alpha^{\tau}\left(\alpha^{\tau+1}+\alpha^{\tau}\left(-\lambda-\eta_{1}\right)\right. \\
& +\alpha^{\tau-1}\left(\lambda \cdot \eta_{1}-\eta_{2}\right)+\alpha^{\tau-2}\left(\lambda \cdot \eta_{2}-\eta_{3}\right) \\
& \cdots+\alpha^{2}\left(\lambda \cdot \eta_{\tau-2}-\eta_{\tau-1}\right) \\
& \left.+\alpha^{1}\left(\lambda \cdot \eta_{\tau-1}-\eta_{\tau}-\varepsilon\right)+\left(\lambda \cdot \eta_{\tau}+\varepsilon\right)\right) .
\end{aligned}
$$

All eigenvalues can be set to zero using $\varepsilon=-\lambda^{\tau+1} /(l-1)$ $\eta_{\tau}=+\lambda^{\tau} /(l-1)$ and $\eta_{i}=-\lambda^{i}$ for $1 \leq i \leq \tau-1$.

The general formulas even for more than one positive Lyapunov exponent or multiparameter control are given in Appendix [C]

\section{CONCLUSIONS}

We have presented methods to improve Poincarésection based chaos control for delayed measurement. For both classes of algorithms, OGY control and difference control, delay affects control, and improved control strategies have to be applied. Improved strategies contain one of the following principle ideas: Rhythmic control, control with memory for previous states, or control with memory for previously applied control amplitudes.

In special cases the unmodified control, previous state memory control, or rhythmic control methods could be considered, especially when experimental conditions restrict the possibilities of designing the control strategy. 
In general, the proposed LPLC and MDC strategies allow a so-called deadbeat control with all eigenvalues zero; and they are in this sense optimal control methods. All parameters needed for controller design can be calculated from linearization parameters that can be fitted directly from experimental data. This approach has also been sucessfully applied in an electronic [13] and plasma 14] experiment.

\section{APPENDIX A: TRANSFORMATION ON THE EIGENSYSTEM}

Here we derive how for one unstable dimension the stabilization problem reduces to the one-dimensional case. Using a covariant basis from right eigenvectors $\vec{e}_{\mathrm{u}}$ and $\vec{e}_{\mathrm{s}}$ to eigenvectors $\lambda_{\mathrm{u}}$ und $\lambda_{\mathrm{s}}$ and the corresponding contravariant left eigenvectors $\vec{f}^{\mathrm{u}}$ and $\vec{f}^{\mathrm{s}}$ of matrix $L$. we can transform the linearized dynamics

$$
\vec{x}_{t+1}=L \vec{x}_{t}+M \vec{r}_{t}
$$

with help of $\mathbf{1}=\vec{e}_{\mathrm{u}} \cdot \vec{f}^{\mathrm{u}}+\vec{e}_{\mathrm{s}} \cdot \overrightarrow{f^{\mathrm{s}}}$ and $L=\lambda_{\mathrm{u}} \vec{e}_{\mathrm{u}} \cdot \vec{f}^{\mathrm{u}}+$ $\lambda_{\mathrm{s}} \vec{e}_{\mathrm{s}} \cdot \vec{f}$. We define as coordinates in the eigensystem $x_{t}^{\mathrm{u}}:=\overrightarrow{f^{\mathrm{u}}} \vec{x}_{t}$ and $x_{t}^{\mathrm{s}}:=\overrightarrow{f^{\mathrm{s}}} \vec{x}_{t}$ giving

$$
\begin{aligned}
& x_{t+1}^{\mathrm{u}}=\lambda_{\mathrm{u}} x_{t}^{\mathrm{u}}+r_{t} \mu_{\mathrm{u}} \\
& x_{t+1}^{\mathrm{s}}=\lambda_{\mathrm{s}} x_{t}^{\mathrm{s}}+r_{t} \mu_{\mathrm{s}}
\end{aligned}
$$

We consider a general ansatz that the control signal is linear in $x_{t-j}$ and $r_{t-i}$,

$$
r_{t}=\sum_{j=0}^{\infty} \vec{K}_{j} \cdot \vec{x}_{t-j}+\sum_{i=0}^{\infty} \eta_{i} \cdot r_{t-i}
$$

Here $r_{t}=\vec{K} \cdot \vec{x}_{t}$ is OGY control. For $r_{t}$ follows

$$
\begin{aligned}
r_{t} & =\sum_{j=0}^{\infty} \vec{K}_{j}\left(\vec{e}_{\mathrm{u}} \cdots \vec{f}^{\mathrm{u}}+\vec{e}_{\mathrm{s}} \cdot \vec{f}^{\mathrm{s}}\right) \vec{x}_{t-j}+\sum_{i=0}^{\infty} \eta_{i} \cdot r_{t-i} \\
& =\sum_{j=0}^{\infty}\left(\vec{K}_{j} \cdot \vec{e}_{\mathrm{u}}\right) x_{t-j}^{\mathrm{u}}+\sum_{j=0}^{\infty}\left(\vec{K}_{j} \cdot \vec{e}_{\mathrm{s}}\right) x_{t-j}^{\mathrm{s}}+\sum_{i=0}^{\infty} \eta_{i} \cdot r_{t-i} \\
& =\sum_{j=0}^{\infty} K_{j}^{\mathrm{u}} x_{t-j}^{\mathrm{u}}+\sum_{j=0}^{\infty} K_{j}^{\mathrm{s}} x_{t-j}^{\mathrm{s}}+\sum_{i=0}^{\infty} \eta_{i} \cdot r_{t-i}
\end{aligned}
$$

If control in stable direction is chosen zero, i.e. $\forall_{j} K_{j}^{\mathrm{s}}=$ 0 , the dynamics decouples in two two systems in stable direction (that is unaffected, but was stable before) and in unstable direction,

$$
x_{t+1}^{\mathrm{u}}=\lambda_{\mathrm{u}} x_{t}^{\mathrm{u}}+\mu_{\mathrm{u}}\left(\sum_{j=0}^{\infty} K_{j}^{\mathrm{u}} x_{t-j}^{\mathrm{u}}+\sum_{i=0}^{\infty} \eta_{i} \cdot r_{t-i}\right) .
$$

This equation is only one-dimensional, so it is sufficient to investigate the one-dimensional control problem if there is only one instable direction. The generalization to higher-dimensional unstable subspaces is straightforward.

\section{APPENDIX B: STABILIZATION OF DELAYED MAPS}

We consider the motion around an unstable fixed point $\vec{x}^{*}$ of the map

$$
\vec{x}_{t+1}=\vec{F}\left(\vec{x}_{t}, \vec{r}_{t}\right)
$$

where $\vec{r}_{t}$ and $\vec{x}_{t}$ have the same dimension of the phase space of the system (although it is desirable to achieve control with a minor number of control parameters). The linear time evoution around the instable fixed point (we choose $\vec{x}^{*}=\overrightarrow{0}$ and $\vec{r}=\overrightarrow{0}$ in the fixed point) is given by Jacobians $D_{x}=: L$ and $D_{r}=: M$

$$
\vec{x}_{t+1}=L \vec{x}_{t}+M \vec{r}_{t}
$$

Delayed measurement means that the applied control $\vec{r}_{t}$ can only be computed from an older value $\vec{x}_{t-\tau}$. Additionally one can store the control amplitudes $\vec{r}_{1} \ldots \vec{r}_{t-\tau}$ applied in the meantime to the system and use the linearization to predict the present position $\vec{x}$ (Linear predictive logging control, LPLC [23]).

Thus we have the general feedback ansatz

$$
\vec{r}_{t}=K \vec{x}_{t-\tau}+\sum_{j=1}^{\tau} N_{j} \vec{r}_{t-j}
$$

Under the condition that $\operatorname{det} M \neq 0$, the feedback can be chosen to

$$
\begin{aligned}
K & =-M^{-1} L^{\tau+1} \\
\forall_{1 \leq i \leq \tau} & N_{i}=-M^{-1} L^{i} M
\end{aligned}
$$

Iterating $\vec{x}_{t}=L^{\tau} x_{t-\tau}+\sum_{j=1}^{\tau} L^{j-1} M \vec{r}_{t-j}$, one has

$$
\begin{aligned}
\vec{x}_{t+1}= & L \vec{x}_{t}+M \vec{r}_{t}=L^{\tau+1} x_{t-\tau}-M M^{-1} L^{\tau+1} x_{t-\tau} \\
& +\sum_{j=1}^{\tau} L^{j-1} M \vec{r}_{t-j}-\sum_{j=1}^{\tau} M M^{-1} L^{j} M \vec{r}_{t-j}=\overrightarrow{0}
\end{aligned}
$$

\section{APPENDIX C: DELAYED MEASURED MAPS - STABILIZING UNKNOWN FIXED POINTS}

Now we show that even fixed points whose exact position is not given (only an approximative value is needed to determine the position of a $\delta$-ball inside which control is switched on) can be stabilized by a difference control method that is similar to the LPLC method given above, even if one has only delayed knowledge of differences of the system variables $x_{t-\tau}-x_{t-\tau-1}$. Combined with stored values of the meantime control amplitudes $\vec{r}_{1} \ldots \vec{r}_{t-\tau-1}$ we propose the control scheme

$$
\vec{r}_{t}=K\left(\vec{x}_{t-\tau}-x_{t-\tau-1}\right)+\sum_{j=1}^{\tau+1} N_{j} \vec{r}_{t-j}
$$


with the feedback matrices

$$
\begin{aligned}
K & =-M^{-1} L^{\tau+2}(L-\mathbf{1})^{-1} \\
\forall_{1 \leq i \leq \tau} \quad N_{i} & =-M^{-1} L^{i} M \\
N_{\tau+1} & =M^{-1} L^{\tau+1}(L-\mathbf{1})^{-1} M
\end{aligned}
$$

Here we have to assume that not only $M$ but also $(L-\mathbf{1})$ is invertible. Using the linear approximation one easily computes directly that this control leads to $\vec{x}_{t+1}=\overrightarrow{0}$, and again this is a so-called deadbeat control scheme where all eigenvalues are zero.

Using the linearized dynamics, we have

$$
\begin{aligned}
\vec{x}_{t-\tau} & =L \vec{x}_{t-\tau-1}+M \vec{r}_{t-\tau-1} \\
\vec{x}_{t} & =L^{\tau+1} \vec{x}_{t-\tau-1}+\sum_{j=1}^{\tau+1} L^{j-1} M \vec{r}_{t-j}
\end{aligned}
$$

and the next iteration reads

$$
\vec{x}_{t+1}=L \vec{x}_{t}+M \vec{r}_{t}=L^{\tau+2} \vec{x}_{t-\tau-1}+\sum_{j=1}^{\tau+1} L^{j} M \vec{r}_{t-j}
$$

[1] Th. Mausbach, Th. Klinger, A. Piel, A. Atipo, Th. Pierre, and G. Bonhomme, Phys. Lett. A 228, 373-377 (1997)

[2] O. J. M. Smith, Closed control of loops with dead time, Chemical Engineering Progress 53, May, 217-219 (1953).

[3] Z.Palmor, Stability properties of Smith dead-time compensator controllers, Int. J. Control 32 (1980).

[4] T. Hägglund An industrial dead-time compensating PI controller, Control Engineering Practice 4, 749-756 (1996).

[5] E. F. Carmacho and C. Bordons, Model Predictive Control, Springer, London (1999).

[6] J. Sieber and B. Krauskopf, Extending the permissible control loop latency for the controlled inverted pendulum, Applied Nonlinear Mathematics Research Report 2004.13, University of Bristol (2004). J. Sieber and B. Krauskopf, Nonlinearity 17(1), 85-103 (2004).

[7] Christopher Edwards, Sarah K. Spurgeon, Sliding mode control: theory and applications. Taylor \& Francis, London (1998).

[8] E. Ott, C. Grebogi and J. A. Yorke, Phys. Rev. Lett. 64, 1196-1199 (1990).

[9] K. Pyragas, Phys. Lett. A 170, 421-428 (1992).

[10] A. W. Hübler, Helv. Phys. Acta 62, 343-346 (1989).

[11] J. E. S. Socolar, D. W. Sukow, and D. J. Gauthier, Phys. Rev. E 50 (6), 3245-3248 (1994).

[12] J. N. Blakely, L. Illing, and D. J. Gauthier, Controlling Fast Chaos in Delay Dynamical Systems, Phys. Rev. Lett. 92, 193901 (2004).

[13] J. C. Claussen, T.Mausbach, A. Piel, and H. G. Schuster, Phys. Rev. E 58, 7256 (1998)

[14] T. Mausbach, T. Klinger, and A. Piel, Chaos and chaos control in a strongly driven thermionic plasma diode,

$$
\begin{aligned}
& -M M^{-1} L^{\tau+2}(L-\mathbf{1})^{-1} L \vec{x}_{t-\tau-1} \\
& -M M^{-1} L^{\tau+2}(L-\mathbf{1})^{-1} M \vec{r}_{t-\tau-1} \\
& +M M^{-1} L^{\tau+2}(L-\mathbf{1})^{-1} \vec{x}_{t-\tau-1} \\
& -\sum_{j=1}^{\tau} M M^{-1} L^{j} M \vec{r}_{t-j} \\
& +M M^{-1} L^{\tau+1}(L-\mathbf{1})^{-1} M \vec{r}_{t-\tau-1}=\overrightarrow{0}
\end{aligned}
$$

By this method the delay can be overcome and even fixed points whose position is known inaccurate can be stabilized from measured differences, provided that one has a sufficient number of control parameters, i. e. $\operatorname{dim} \vec{r}=\operatorname{dim} \vec{x}$, and both $M$ and $(L-\mathbf{1})$ are invertible.
Physics of Plasmas 6 (10), 3817-3823 (1999).

[15] S. Bielawski, D. Derozier, and P. Glorieux. Phys. Rev. A, 47 (4), 2492-2495, (1993).

[16] W. Just, T. Bernard, M. Ostheimer, E. Reibold, and H. Benner, Phys. Rev. Lett. 78, 203 (1997).

[17] W. Just, E. Reibold, K. Kacperski, P. Fronczak, and J. Hołyst, Phys. Lett. A 254, 158 (1999).

[18] G. Franceschini, S. Bose, and E. Schöll Phy. Rev. E 60, 5426 (1999)

[19] W. Just, D. Reckwerth, E. Reibold, and H. Benner, Phy. Rev. E 59, 2826 (1999).

[20] Philipp Hövel and Joshua E. S. Socolar, Phy. Rev. E 68, 036206 (2003).

[21] W. Just, S. Popovich, A. Amann, N. Baba, and E. Schöll, Phys. Rev. E 67, 026222 (2003).

[22] J. C. Claussen, nlin.CD/0204031 Phys. Rev. E, accepted for publication (2004).

[23] J. C. Claussen, PhD thesis (in german), Kiel (1998).

[24] J. C. Claussen, Control of delayed measured systems and impulse length limitations in difference control, in: Alexander L. Fradkov (ed.): Proceedings PHYSCON 2003, St. Petersburg, Vol. 4, pp.1296-1302 (2003).

[25] J. K. Hale, S. M. Verduyn Lunel, Introduction to Functional Differential Equations, Springer, New York (1993).

[26] J. C. Claussen, nlin.CD/0204060

[27] H. G. Schuster and M. B. Stemmler, Phys. Rev. E 56 (6), 6410-6417 (1997)

[28] Valery Petrov and Kenneth Showalter: Phys. Rev. Lett. 76, 3312-3315 (1996) and references therein.

[29] J. E. S. Socolar, D. J. Gauthier, Phys. Rev. E 57 (6), 6589-6595 (1998). 